\title{
Spin and activity of comet 67P/Churyumov-Gerasimenko
}

\author{
J.-B. Vincent ${ }^{1}$, L. M. Lara ${ }^{2}$, G. P. Tozzi ${ }^{3}$, Z.-Y. Lin ${ }^{4}$, and H. Sierks ${ }^{1}$ \\ 1 Max-Planck-Institut für Sonnensystemforschung, Max-Planck-Strasse 2, 37191 Katlenburg-Lindau, Germany \\ e-mail: vincent@mps.mpg.de \\ 2 Instituto de Astrofísica de Andalucía, CSIC, PO Box 3004, 18080 Granada, Spain \\ 3 INAF - Osservatorio Astrofisico di Arcetri, Largo E. Fermi 5, 50125 Firenze, Italy \\ ${ }^{4}$ Institute of Astronomy, National Central University, Taiwan
}

Received 5 April 2012 / Accepted 6 November 2012

\section{ABSTRACT}

\begin{abstract}
Aims. In 2014 ESA's spacecraft Rosetta will reach its final target: comet 67P/Churyumov-Gerasimenko (67P). Although this comet has been studied in great detail over the past decade, there are still some uncertainties regarding the actual spin orientation and the distribution of active regions on the surface. We here give an overview of our current knowledge of these critical parameters based mainly on data acquired during the last orbit of the comet aims to deliver a review of what is our current knowledge of these critical parameters.

Methods. We based our analysis on the modeling and interpretation of dust jets seen in the coma of 67P during the 2009 apparition. Results. The inversion of ground-based images leads to an estimate of the spin axis orientation $\left(\mathrm{RA}=40^{\circ} \pm 10^{\circ}, \mathrm{Dec}=70^{\circ} \pm 10^{\circ}\right.$ ) and to the localization of the most prominent active areas on the surface of the nucleus. We can reproduce the activity with three active regions localized at $-45^{\circ}, 0^{\circ}$, and $+60^{\circ}$ of latitude. We use these results to revisit the observations taken during the 2003 apparition and examine the changes in activity between then and now. Our model from 2009 is also valid for the 2003 data, which suggests that no significant change in spin orientation and active areas occurred from one perihelion passage to the next. Finally, we combine all the results to propose some predictions on the activity to be expected in 2015 during the comet exploration phase of the Rosetta mission.
\end{abstract}

Key words. methods: numerical - comets: general - comets: individual: 67P/Churyumov-Gerasimenko

\section{Introduction}

ESA's spacecraft Rosetta is now on its final cruise toward comet 67P and will reach its target in 2014. The spacecraft will follow the comet as it returns to perihelion, monitoring the activity and releasing a lander to study the surface in situ. To be ready for the final stage of the mission, many observations of the comet have been acquired during the last orbits of 67P, but some uncertainties still remain. Several authors have published different solutions for the spin axis orientation, for instance, and the shape of the comet is not yet well constrained. A good review of the current knowledge about these nucleus parameters can be found in Lamy et al. (2006) and Lamy et al. (2007). These authors present an in-depth comparison between different solutions for the spin axis orientation, which seems to cluster in two regions of the RA/Dec plane that vary depending on if the rotation is prograde or retrograde. We discuss below whether our estimation agrees well with that was presented by these authors, and how the dynamical simulations allow the proposed solution to be refined.

Lara et al. (2011) have shown that the coma of 67P exhibits several dust jets, which indicates that the activity is not isotropically distributed on the surface, but rather enhanced in specific regions of the nucleus. Because these dust jets can potentially be a serious hazard for the spacecraft, especially for the lower orbital phases of the mission, we need to constrain them better. We aim to provide an estimate of the activity pattern that is expected during the mission, the directions of the jets, and the sizes and velocities of the dust particles involved. Over the past four years we have developed a model of dust coma structures, aiming at simulating the behavior of dust jets from the subsurface of cometary nuclei up to thousands of kilometers away from the surface. Our model was used successfully to study several targets: 73P/Schwassmann-Wachmann 3 (Vincent et al. 2010a), 9P/Tempel 1 (Vincent et al. 2010b), and 81P/Wild 2 (Lin et al. 2012). For the last two targets, we have reproduced the behavior of the jets from ground-based observations only, with results that agree with in situ measurements by the Deep Impact and Stardust spacecrafts.

\section{Data and model}

We applied the same method to two sets of images of $67 \mathrm{P}$ acquired in 2008-2009, before and after perihelion passage. At that time the comet was very active and showed several dust coma structures that we analyzed to localize the active regions at the surface of the nucleus, and to constrain the properties of the dust grains in the jets. Details on the observations and basic data reduction, along with the analysis of dust and gas production rates, can be found in Tozzi et al. (2011) and Lara et al. (2011). For our analysis flux-calibrated and flat-fielded/bias-subtracted images of the comets were used.

\subsection{Data}

Comet 67P reached perihelion on 28.433 February 2009. The active part of the inbound approach was monitored from 01 June 2008 to 13 January 2009, i.e., from 2.98 AU to 1.39 AU. Images were acquired at the $8.2 \mathrm{~m}$ Unit Telescope of Very Large Telescope (VLT) at the European Southern Observatory in Chile (program Ids 381.c-0123 and 082.C-0740), using the FORS2 focal reducer instrument and with the Telescopio Nazionale Galileo (TNG) at La Palma in Spain, using the camera-spectrograph DOLORES. Series of images were taken with Bessell VRI broadband filters. The contribution of light from gas and ion emissions in these images is negligible with respect to the dust contribution (Tozzi et al. 2011). 
From 22 February 2009 to 16 April 2009 the comet was monitored from the Calar Alto Observatory (CSIC-MPG, near Almeria, Spain). In addition, it was observed at Lulin Observatory (National Central University, Taiwan) from 25 January 2009 until 22 March 2009. We focus on images in broadband Johnson R filters, mainly acquired with the CAFOS instrument mounted on the $2.2 \mathrm{~m}$ telescope at Calar Alto. A preliminary description of the coma morphology for this period can be found in Lara et al. (2011). We expand this analysis in the next section of the paper.

\subsection{Image enhancement and detection of features}

In both datasets, no coma structure can be directly detected in the reduced images. This is a common problem in coma morphology analysis, and arises because the signal intensity of such structures is barely above the coma background, which itself has a strong radial gradient toward the nucleus position; it is thus difficult for our eyes to notice the differences caused by the presence of jets. Hence we processed the images with specific numerical filters designed for enhancing inherent structures, mainly by removing the coma background signal. Filtering techniques have been used for decades and filters are constantly refined. However, it is necessary to proceed with caution when interpreting the filtered images for two main reasons: (1) The processing can destroy the photometric information contained in the image. Hence, a brighter structure seen in the filtered image does not necessarily mean that more dust was present in this jet. (2) Each filtering technique can introduce artifacts in the result image, some of them almost indistinguishable from a real dust feature. It is therefore very important to always cross-check a detection by comparing the outcome of independent filtering techniques (for instance a Laplace derivative of the image and an unsharp masking). Despite the difficulties, it is still necessary to look for possible signatures of the coma structures in the original images after the features were identified in the enhanced data. Although the features themselves are not visible, for a trained eye the distorted coma indicates their presence. For reference, exhaustive comparisons of image enhancing techniques for dust coma morphology can be found in Schwarz et al. (1989), Larson \& Slaughter (1992), Schleicher \& Farnham (2004), Vincent (2010) and, Samarasinha \& Larson (2011).

The general procedure to identify dust coma features comprises several steps: we processed all images with at least three different techniques: Larson-Sekanina (rotational-difference) filtering (Larson \& Sekanina 1984), radial normalization (A'Hearn et al. 1986), and adaptive Laplace filtering (Boehnhardt \& Birkle 1994). An independent verification was also made by looking for anisotropies in the isophotes to see if they correlated with the outcome of the filtering. In enhanced images, structures are identified as long, narrow features. They often extend radially from the nucleus location and can be bent in the antisolar direction. In some cases, mostly when the spin axis of the nucleus points toward the observer, the dust features can be observed as arcs or spirals in the coma (see Sekanina 1987, for a complete description of the influence of viewing geometry). We followed the evolution of every detected feature in all images to analyze its repeatability. A first quick analysis can be made by comparing the changes in the geometry of the structures with the changes in viewing geometry if the spin axis orientation and rotation period are known. In parallel we also simulated the dust tail and trail using the model developped by Beisser \& Drechsel (1992). This allowed us to clearly distinguish these features from coma jets.

\subsection{Activity model}

Modeling the activity is not trivial because the mechanism behind the formation of dust jets is still unknown, although several models have already been proposed. The current explanations assume either an active area at the surface (Sekanina 1987), a collimation of the global gas flow by local topographic features such as concavities (Crifo \& Rodionov 1997), or a combination of both effects. It has been shown, though, that most of the very collimated features such as thoses described in this paper can be explained by the presence of active regions on the surface, which rotate with the nucleus. For several in-situ missions and comets, Farnham et al. (2007, 2012), Vincent et al. (2010b), and Lin et al. (2012) could follow the evolution of the dust jets and connected them unambiguously to specific regions of the surfaces without requiring large-scale gas flows and a specific topography to constrain the jets. Although we understand the importance of the nucleus concavities on the global flows and broad features observed in the coma (for instance Szegö et al. 2002 on jets of comet $1 \mathrm{P} /$ Halley), they are not needed to explain the narrow, collimated structures described here.

An exhaustive review of the processes taking place in the active areas can be found in Belton (2010). However, a full understanding of the origin of these collimated outflows is not required to perform simulations at the scale of our observed images. As we have shown in Vincent et al. (2010b) and Lin et al. (2012), good approximations of the initial size and velocity distributions of the particles are sufficient to fully reproduce the behavior of the jets. Thus our model does not simulate the initial acceleration phase of the dust particles when they are lifted from the surface and accelerated by the gas. The model assumes that the motion of the dust grains can be decoupled from the gas, which is typically the case after a few radii from the surface, far below the spatial resolution of our images. At this stage, the dust size distribution is well described with a decreasing power law:

number of particles of size $d=\left\{\begin{array}{cl}d^{-\alpha} & \text { if } d_{\min } \leq d \leq d_{\max } \\ 0 & \text { elsewhere }\end{array}\right.$

Here, $\alpha$ can be measured from astronomical images (Fulle 1999; Markovich \& Markovich 2001; Min et al. 2005) and its values usually ranges between 2.5 and 3.0 for grains with a size varying from micrometer to millimeter. The terminal velocity reached by the particles after acceleration is given by Fulle (1987)

$v=v_{0} \beta^{1 / 6} \quad\left(\mathrm{~m} \mathrm{~s}^{-1}\right)$,

where $v_{0}$ is constant for a given heliocentric distance. $\beta$ is here the ratio between solar gravity and solar radiation pressure as described by Finson \& Probstein (1968). For particles larger than a micrometer, it can be approximated as a function of the grain size:

$\beta \simeq \frac{k}{d}$

where $k$ is a constant depending on the material. For cosmically relevant material, Burns et al. (1979) showed that $k=4 \times 10^{-7}$ is a good approximation if the composition of the grains is unknown.

The initial velocity direction is assumed to be perpendicular to the convex hull of the surface. This implies that the sources are finally localized on the surface of the convex topological envelope, which may or may not coincide with the real surface, and a detailed hydrodynamical model of the acceleration zone 

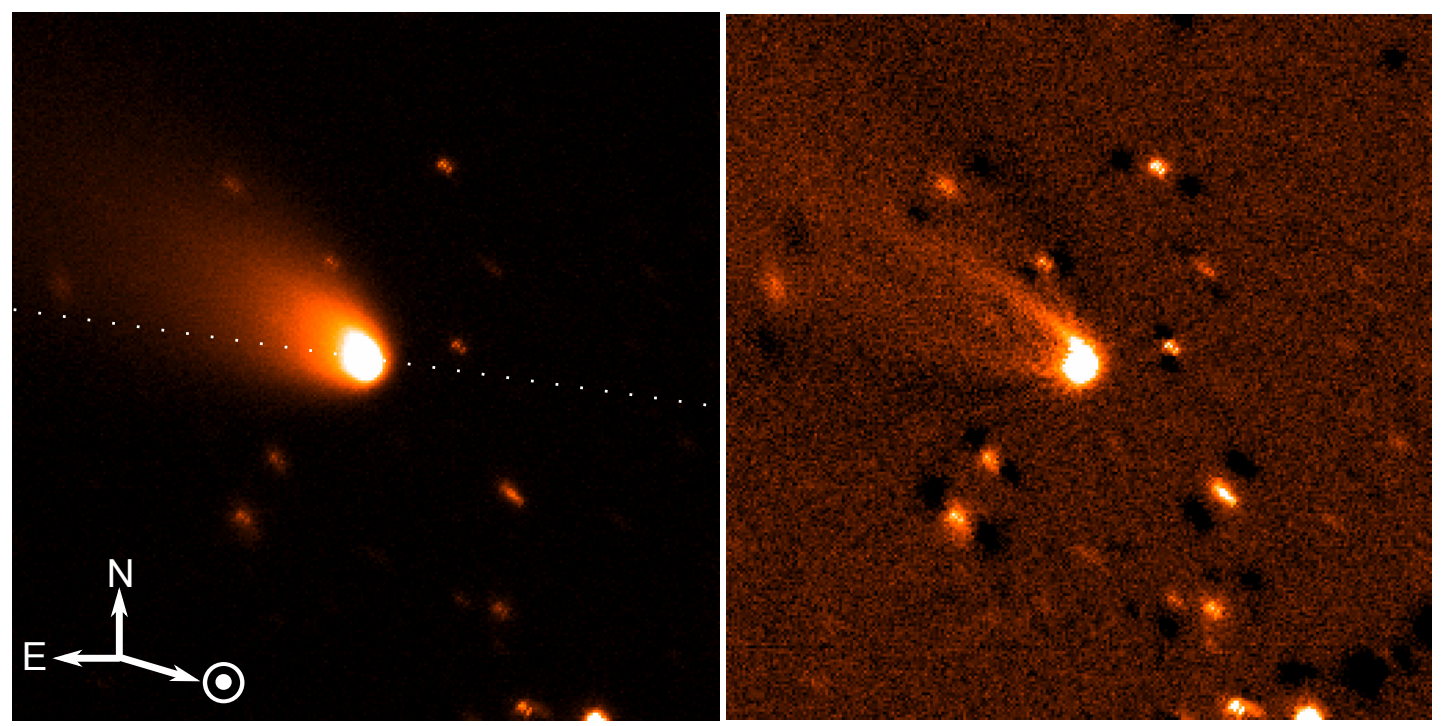

Fig. 1. Dust coma of 67P in the pre-perihelion epoch. The left panel shows an image of the dust coma acquired on 22 Oct. 2008, at heliocentric distance of $1.90 \mathrm{AU}$. Orientation: north is up and east is left. Field of view: $195 \times 195 \mathrm{arcsec}=273000 \mathrm{~km}$ at the comet. The right panel is the same image processed with a Larson-Sekanina filter. The dotted line traces the orbital plane of the comet.
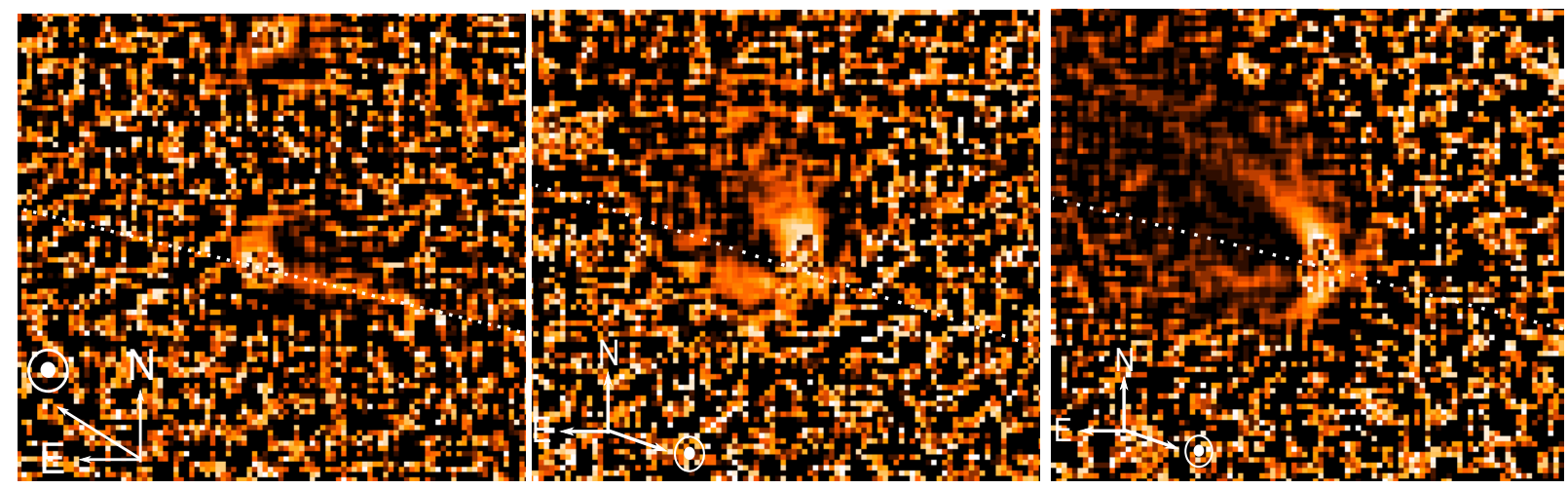

Fig. 2. Dust coma of 67P in the pre-perihelion epoch. Broadband $R$ images acquired on 1 Jun. 2008, 8 Sep. 2008, 22 Oct. 2008 (Tozzi et al. 2011 ) and numerically enhanced with the adaptive Laplace filter. Field of view: $100 \times 100$ arcsec $=210000 \mathrm{~km}, 160000 \mathrm{~km}$, and $135000 \mathrm{~km}$ at the comet. Some features are detected in the coma, although not with every image-processing technique. The salt-and-pepper pattern represents the noise structure of the background, with embedded stars trails. The dotted line traces the orbital plane of the comet.

must be taken into account to identify the sources more precisely. However, our previous studies (Vincent et al. 2010b; Lin et al. 2012) have demonstrated that this approximation is valid for most cases where one tries to invert the location of active sources from ground-based images. Furthermore, recent work that took into account the full gas flows over complex topographies through direct Monte Carlo simulations has validated this claim (Vincent et al. 2012). Only for very rapid changes of the local slope (for instance the cliffs of 9P/Tempel 1) could we see a deviation from the perpendicular direction, although not by more than 20 degrees.

The simulation was carried out with the computer code COSSIM (COma Structure SIMulator) which performs a full 3D integration of the motion of dust particles after they are ejected from an irregularly shaped nucleus. The model takes into account the rotation of the nucleus, local illumination, solar gravity, and radiation pressure. It outputs $3 \mathrm{D}$ positions of the particles after a given time, and a simulation of how the dust jets would look like from a specific view point of observation (either from Earth or a spacecraft). As stated above, in this version of the model the interaction between gas and dust at close distance from the surface is not directly simulated, but implicitly described in the laws that state the initial size and velocity distributions. All details of the model are published in Vincent et al. (2010b).

\section{Coma morphology}

\subsection{Between 3 and 2 AU before perihelion}

Images acquired in the pre-perihelion phase 2008 and 2009 do not display unambiguous coma structures until about a month before perihelion. The dust coma expands slowly and reaches large distances in antisolar direction as the comet approaches the Sun, from 2.98 AU in June 2008 to 1.36 AU in January 2009. Figure 1 exemplifies the appearance of the coma during this period. The isophotes present few distortions apart from an extension in tail direction. No outbursts or evidence of dust jets in the coma could be detected by inspecting the flux-calibrated images and the respective isophote patterns. However, adaptive Laplace filtering reveals some anisotropies in the tail direction. Because we did not detect these patterns with other filters such as Larson-Sekanina or unsharp masking, one could consider them artifacts due to the filtering, but it could also be that their level of signal is too close to the background for them to be enhanced by traditional filters. Figure 2 shows some of the 

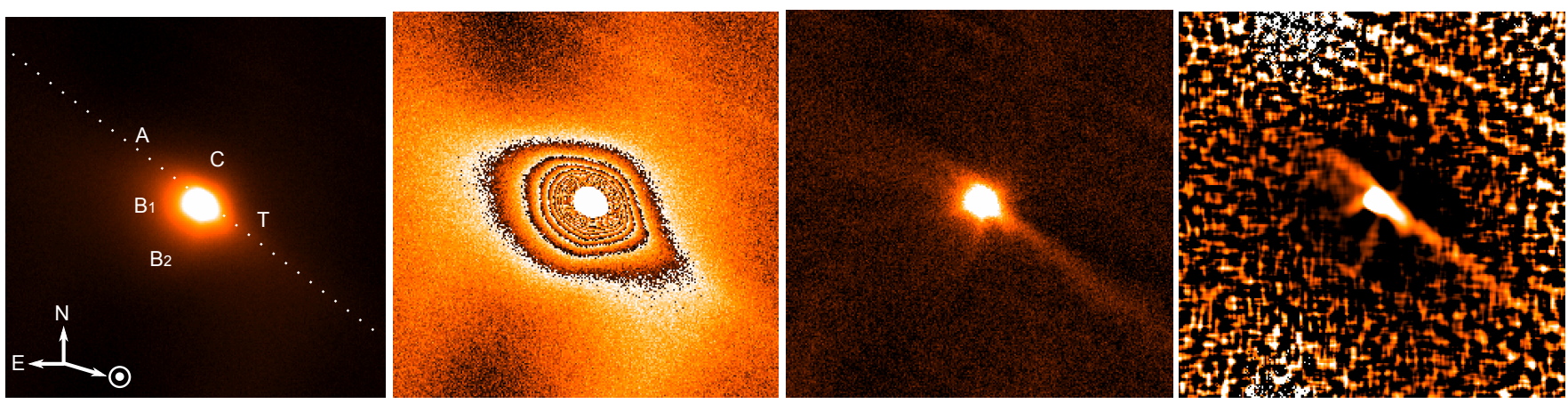

Fig. 3. Dust coma of 67P shortly before perihelion. Image acquired on 18 Feb. 2009, at heliocentric distance of 1.25 AU. Orientation: north is up and east is left. Field of view: $106 \times 106$ arcsec $=128600 \mathrm{~km}$ at the comet. The same acquisition is presented through different numerical filters. From left to right: no processing, isophotes, Larson-Sekanina filter, adaptive Laplace filter. The dotted line in the left traces the orbital plane of the comet.

Table 1. Summary of observing epochs used and relevant orbital elements: days from perihelion, helio- and geo-centric distances, true anomaly, phase angle ("Sun-target-observer" angle).

\begin{tabular}{l|ccccccc|ccccc}
\hline \hline Date & $\Delta T_{P}$ & $r(\mathrm{AU})$ & $\Delta(\mathrm{AU})$ & $v^{\circ}$ & ${\mathrm{S}-\mathrm{T}-\mathrm{O}^{\circ}}^{\circ}$ & PsAng $^{\circ}$ & PsAMV $^{\circ}$ & $A^{\circ}$ & $B_{1}^{\circ}$ & $B_{2}^{\circ}$ & $T^{\circ}$ & $C^{\circ}$ \\
\hline 2009-Jan.-25 & -34 & 1.315 & 1.671 & 329.9 & 36.1 & 67.8 & 238.0 & 52 & 155 & 214 & 235 & 320 \\
2009-Jan.-30 & -29 & 1.297 & 1.670 & 334.0 & 36.1 & 67.5 & 237.7 & 42 & 124 & 180 & 231 & ND \\
2009-Feb.-18 & -10 & 1.253 & 1.673 & 350.6 & 35.9 & 67.2 & 238.2 & 55 & 120 & 165 & 235 & 300 \\
2009-Feb.-26 & -2 & 1.246 & 1.680 & 357.8 & 35.8 & 67.5 & 239.1 & 53 & 110 & 170 & 239 & 300 \\
2009-Mar.-12 & +12 & 1.254 & 1.707 & 10.5 & 35.1 & 69.0 & 241.9 & 80 & 160 & 220 & 240 & ND \\
2009-Mar.-19 & +19 & 1.267 & 1.729 & 16.7 & 34.6 & 70.2 & 243.9 & 80 & 160 & 220 & 242 & ND \\
2009-Apr.-11 & +42 & 1.345 & 1.844 & 35.8 & 32.0 & 76.0 & 252.5 & 100 & 170 & 230 & 257 & ND \\
\hline
\end{tabular}

Notes. PsAng indicates the position angle of the extended Sun-comet vector. PsAMV gives the position angle of the anti-velocity vector. Columns $A, B_{1}, B_{2}, T, C$ are the position angles at the coma center of all structures identified in our images, $T$ being the dust trail. The given position angles refer to the center lines of the structures and have an uncertainty of a few degrees. North $=0^{\circ}$, east $=+90^{\circ}$. Structures are sorted by increasing position angle. See Fig. 5 for reference. ND = not detected unambiguously.

Laplace filtered images. The patterns are found on images obtained on 1, 4, 5 June, 4, 6, 8 September, and 22,23, 26 October when the comet approached the Sun from 3 to 2 AU. Because of the uncertainty in the detection, we did not consider these features in the initial analysis. We will revisit these images below to see whether the activity model derived from the images around perihelion could explain what we observed or if they are only processing artifacts.

From the end of January 2009 onward, the coma morphology starts to change; isophotes get distorted, and image enhancement techniques reveal a long spike in southwest direction. At least two dust jets are seen in the enhanced images, but they are quite faint (see Fig. 3). Table 1 summarizes the detections.

\subsection{Around perihelion and post-perihelion}

The dust features become more prominent about ten days before perihelion, and in the outbound phase of the orbit. Apart from the dust tail, up to five structures are unambiguously detected in the images. Four of them $\left(A, B_{1}, B_{2}\right.$, and $C$ in Fig. 5) are typical of dust jet activity, i.e., what we see in the coma is the signature of a dust-emitting region at the surface of the rotating nucleus, as stated by Sekanina (1987). We show in the next section that two of the structures $\left(B_{1}\right.$ and $\left.B_{2}\right)$ are linked to the same active area of the surface, hence the use of the same letter $B$ in the label. Feature $T$ is very different. It appears in our images as a long and narrow spike and does not show any short-term variation that could be associated to the rotation of the nucleus. Its position angle in the images is exactly along the anti-velocity vector of the comet and changes only with the geometry of our observations. Therefore we interpret this spike as the signature of the dust trail in the images. Since neither phase angle nor position angles of the extended Sun-comet radius vector are changing noticeably in this period of time, the changes in coma morphology are not due to a different viewing geometry, but intrinsic to the variability in the cometary dust coma/nucleus activity.

These changes are in more detail:

- Structure A is most prominent. It is seen in all our images and can be detected easily with all filters; it extends on both sides of the nucleus, but is more easily identified in the antisolar direction. In the other direction its falls close to the dust trail and is only seen close to the nucleus (See Fig. 4).

- Structure B consists of two dust features expanding towards the southeast, and is detected in all our images. These structures are interpreted as the edges of a dust cone as explained with the simulations of Sect. 4.2, i.e., the result of dust emission from a single source at the surface of the rotating nucleus. The motion of this cone from January to April 2009 is used by our inversion code to determine the spin axis orientation.

- Structure C is very faint, and one can argue whether it is real or not. Because we detected it in several images and with completely different processing techniques, we are confident that there is indeed a jet at this position angle. The apparent weakness of the jet with respect to the other ones can also be justified, we will discuss it below.

This description is consistent with what other authors reported for previous perihelion passages. Since this comet is known, it has always shown an asymmetric coma after perihelion, with several jets and a very distinct trail. A review of these observations can be found in Lara et al. (2011). 

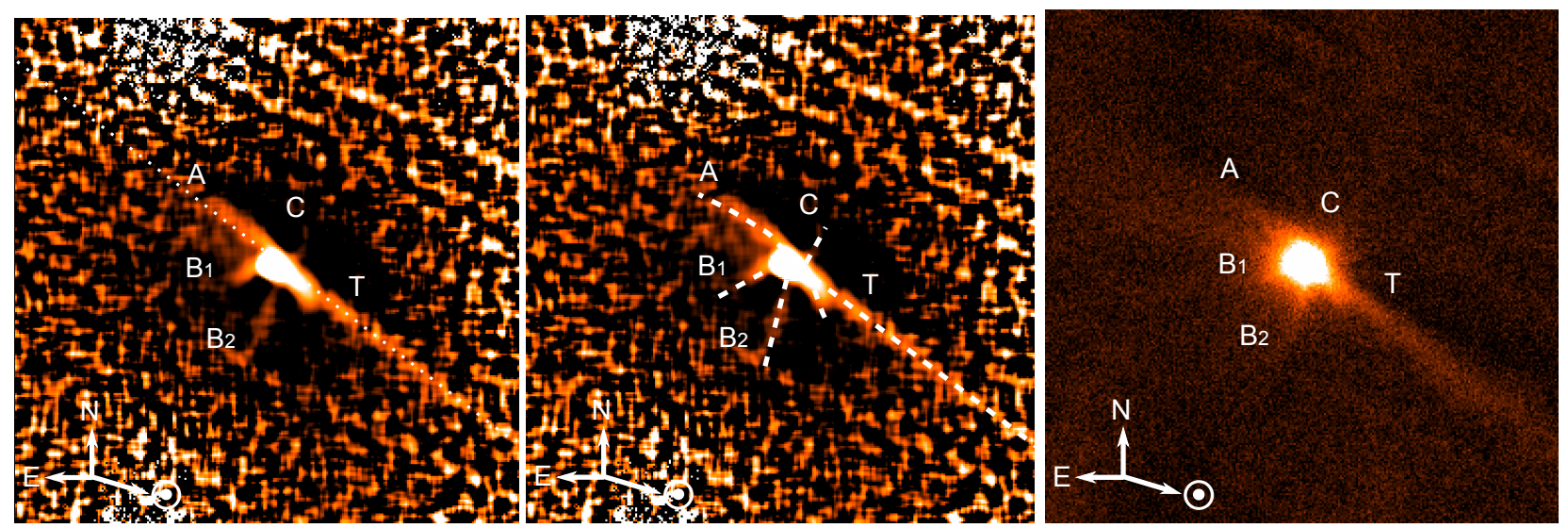

Fig. 4. Dust coma of 67P shortly before perihelion. Image acquired on 18 Feb. 2009, at heliocentric distance of 1.25 AU. Orientation: north is up and east is left. Field of view: $106 \times 106$ arcsec $=128600 \mathrm{~km}$ at the comet. We highlight here the location of the dust coma structures. From left to right: adaptive Laplace-filtered image, adaptive Laplace-filtered image with the structure geometry indicated by dashed lines, Larson-Sekaninafiltered image. The dotted line traces the orbital plane of the comet.

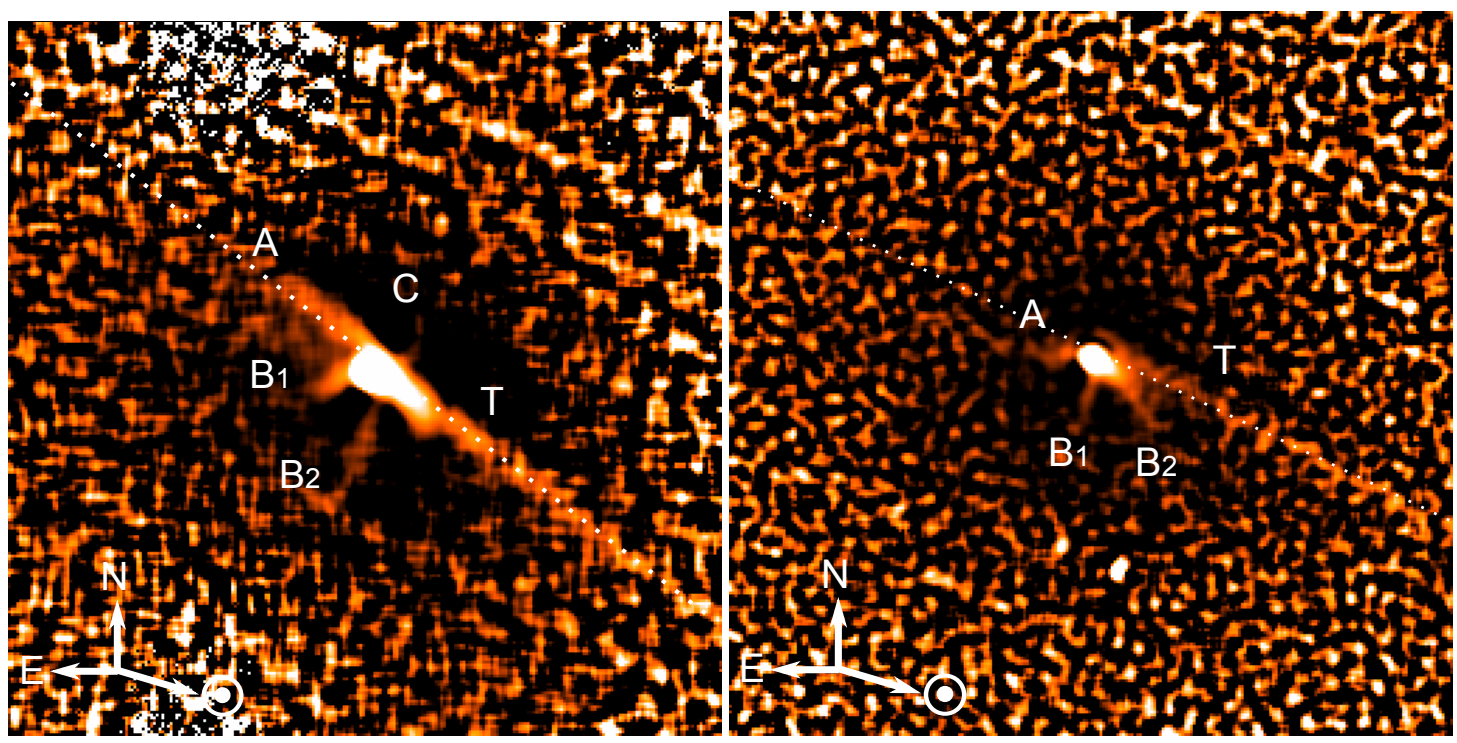

Fig. 5. Coma structures of 67P as observed on 18 February (left) and 19 March (right) 2009 ( -10 days and +18 days from perihelion), Labels indicate the dust jets. $A$ is the most prominent, jets $B$ are the two edges of a cone created by the rotation of the nucleus. $C$ is faint and not detected anymore after perihelion. The dotted line traces the orbital plane of the comet.

\section{Modeling results}

\subsection{Inversion of the spin axis orientation}

The spin axis orientation of the nucleus is a critical parameter for simulating the activity, and on a general scale for the whole Rosetta mission. It controls the illumination of the surface at different latitudes and whether areas can be active or not. Unfortunately, this orientation is not known yet with the best accuracy. Several authors have published determinations of the nucleus orientation based on light curve inversion or the study of the non-gravitational forces. An exhaustive review of all previous determinations was published by Lamy et al. (2006). We revisit this problem through the coma structure modeling approach, and discuss how our results fit in to the existing picture.

The influence of the spin axis orientation on the coma structures was first described by Sekanina (1987). As the nucleus rotates, the dust is emitted along the surface of a cone usually centered on the spin axis. When we observe a jet from far away, we integrate all particles along our line of sight. This enhances the edges of the dust cone, making them more easily detectable. The shape of the structures in our images will depend also on the observing geometry, i.e., the inclination of the spin axis with respect to our line of sight. If we observe a cone sideways, we detect the edges as straight lines in our images, whereas observing it along the spin axis would lead to a spiral or to arcs in the pictures.

Another point to consider is the fact that images are only a $2 \mathrm{D}$ representation of a $3 \mathrm{D}$ phenomenon. With a single observation one can only know the projected pattern the structures form on our plane of sky. Therefore it is always important to monitor the comet for several months to obtain images from different viewing angles to be able to reconstruct the missing third dimension.

The way we obtain a spin axis orientation from coma structures images is described in details in Vincent et al. (2010a) but we summarize it here briefly. Each image is processed with different techniques to reveal coma structures. Based on our understanding of the patterns, an estimate is made for the projected position angle of the spin axis in each image. This angle is then inverted for the whole set of images by testing which of all possible spin axis orientation would lead to this specific position angle for the respective observing epoch. A good fit is defined 


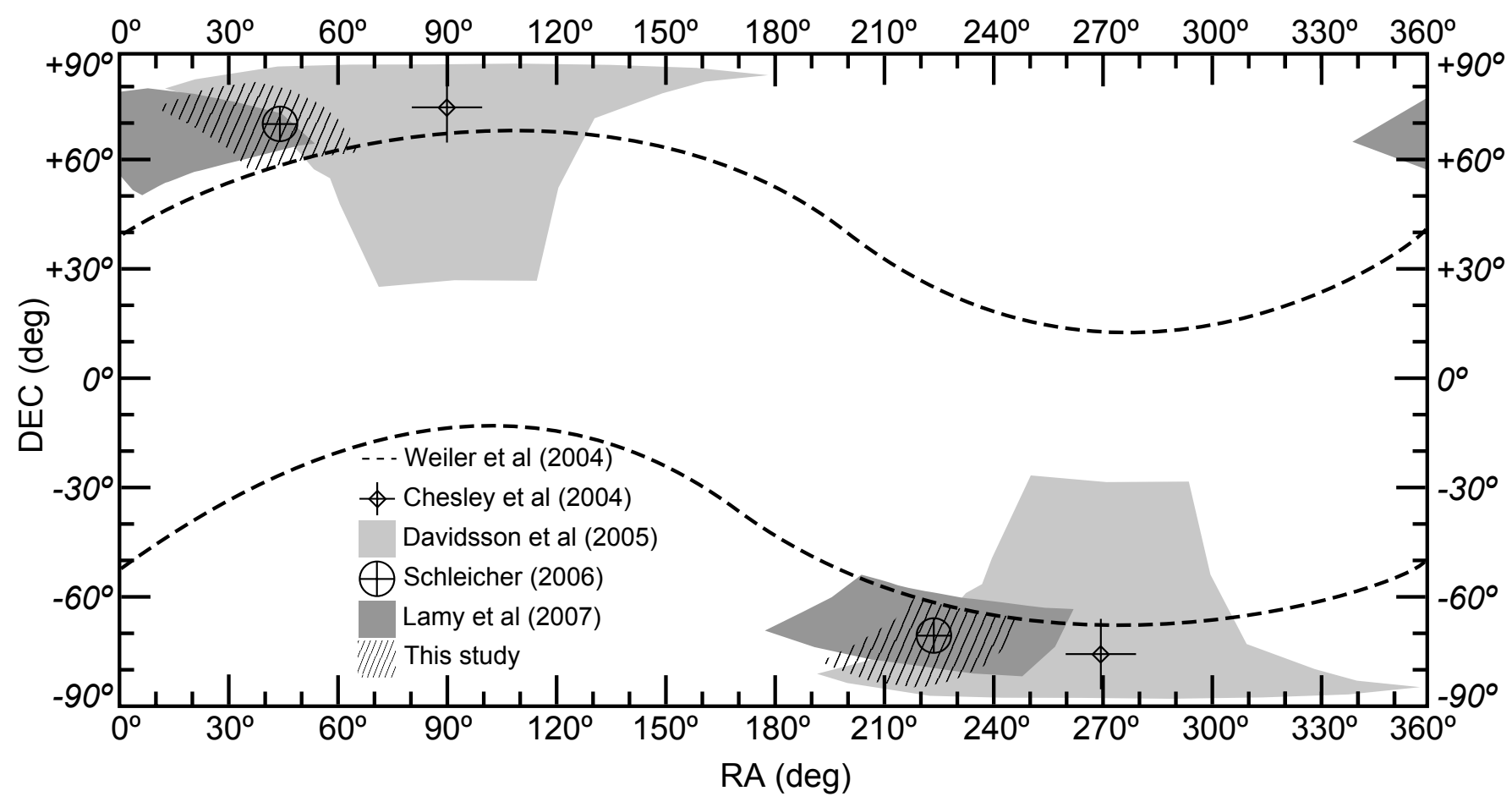

Fig. 6. Review of the spin axis orientation as defined by several authors. These solutions were obtained from different data sets and analyses, yet they cluster in the same regions of the [RA, Dec] space. Weiler et al. (2004): dust jets from 2003; Schleicher (2006): dust jets from 1996; Chesley (2004): modeling of non-gravitational forces; Davidsson \& Gutiérrez (2005): study of the non-gravitational force vector due to nucleus outgassing, based on two different thermophysical models; Lamy et al. (2007): lightcurve inversion; this study: dust jets from 2009.

by a least-squares minimization of the differences between observed and predicted PA of the projected spin axis for all images. If no solution can be found, we must reconsider our initial assumptions and our understanding of the patterns in the images. If a good fit is found, we have a possible spin axis to be tested further with a dust jet simulation.

The initial assumption used in this study was to consider structures $B_{1}$ and $B_{2}$ forming a single emission cone, as generated from the same source on the nucleus forming a single emission cone. We then estimated the projection of the spin axis as similar to the PA of the central axis between $B_{1}$ and $B_{2}$. This estimate was repeated for all images in our set (Table 1). To be complete, we also tried to estimate the spin orientation using different assumptions, for instance treating $B_{1}$ and $B_{2}$ as independent sources. We could not find any axis orientation that would be compatible with the different geometries of observations for all epochs. Even assuming a changing spin orientation and exploring all dust parameters (size distribution, velocities, initial direction of emission) we could not find a consistent solution for the location of the sources.

Using our initial hypothesis of one single source for the southern jets, we found a unique orientation of the spin axis compatible with all observed features. This best orientation is given by the following right ascension (RA) and declination (Dec):

- $\mathrm{RA}=40^{\circ}, \mathrm{Dec}=70^{\circ}$ (prograde rotation)

- $\mathrm{RA}=220^{\circ}, \mathrm{Dec}=-70^{\circ}$ (retrograde rotation $)$,

all values $\pm 10^{\circ}$.

The mean of the difference between observed and simulated PA is only 3.0 degrees. However, we have to add to this the uncertainty in the geometry of the structures. We are looking at extended features that can be partially destroyed by the processing techniques. Usually, the position angle is measured for the imaginary central line of the structure. Different processing techniques will affect the edges of the dust feature differently and not necessarily symmetrically. This will lead to an uncertainty of a few degrees on the PA of each feature in the processed images. Therefore, we estimate the error to be more on the order of 10 degrees on the spin axis orientation.

Our analysis of the coma structures during the post apparition of $67 \mathrm{P}$ did not reveal any alternative solution of similar quality to the one mentioned above. This is demonstrated below when we show the results of the dust jet simulations.

This result was checked against the published values for $67 \mathrm{P}$ (Lamy et al. 2006, 2007) and we found a good agreement with our solutions (Fig. 6). It is important to note that all solutions were obtained using different techniques, perihelion passages, and level of activity. They all cluster in the same areas of the (RA, Dec) map, which means that there is little variation in the spin axis orientation over the years, i.e., the nucleus is more or less in a stable state of rotation. This could be of a great advantage for the in-situ operations of the Rosetta spacecraft. If the nucleus continues to behave in the same way as in the past, we do not expect changes of the spin orientation during the course of the mission, although some level of precession (within our $10^{\circ}$ error bar for the spin axis orientation) cannot be ruled out.

\subsection{Modeling the activity}

The second step of the analysis is to use the spin solution obtained in Sect. 4.1 with the COSSIM code for an attempt to reproduce the coma structures for each observing epoch. The code simulates the dynamics of dust grains in active regions from the surface of the nucleus up to hundreds of thousands of kilometers away. It has been used successfully to reconstruct the activity of 9P/Tempel 1 and 81P/Wild 2, in good agreement with in-situ 

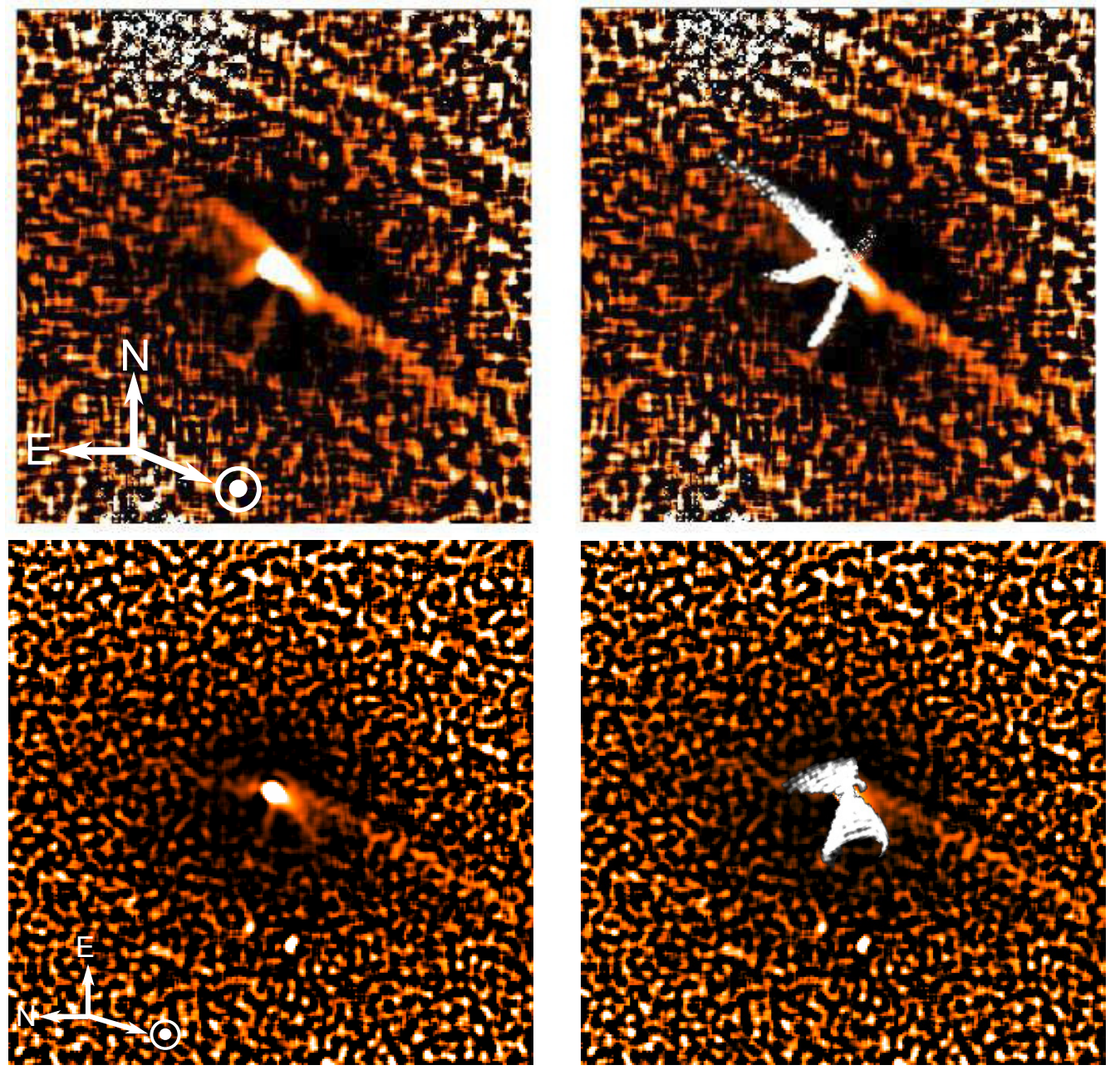

Fig. 7. Model of dust jets for 18 Feb. 2009 (top row) and 19 Apr. 2009 (bottom row). For each date, the left panel is a Laplace-filtered image of $67 \mathrm{P}$ and the right panel the same image with a simulations shown as a white overlay. The model reproduces the shape and direction of the jets. The trail was not included in the simulation.

measurements. Active regions are defined at the surface of the nucleus, which can have a complex 3D shape (if a model is available). For 67P no such a model exists. A possible shape has been proposed by Lamy et al. (2007) from the inversion of a very limited set of lightcurves, but would introduce too many uncertainties in our simulations. Indeed the complete model of the jets, starting from the surface, requires a good understanding of the local topography, as it affects the local illumination conditions, temperature, and gas flows. Because of the large uncertainties on the existing shape model, we restrained our study to an ellipsoid approximation of the nucleus with the size $4.5 \times 3.8 \times 3.4 \mathrm{~km}$ (Lamy et al. 2007). This is the best topological envelope available to describe the surface and is valid as a first approximation. However, since we use the shape to define the original direction of emission, the choice of an ellipsoid vs. a sphere or a real model affects the final localization of the sources. For 67P, the ratio between short and long axis equals 0.75 . For a given direction of emission, this can change the source location by at most 12 degrees with respect to the sphere approximation. This error is comparable to the general uncertainty we have on the location. The definition of the long axis orientation as a function of time is taken from Lamy et al. (2007).

Given these uncertainties on the real shape, our model can only provide some constraints on the latitude of the sources on the topological envelope. The longitude for instance can only be defined if we precisely know the phasing of the nucleus and the local topography. Only then we can link the observed daily activity with temperature profiles and derive a more precise location for the source.

With this approach we could reproduce the observations assuming the presence of three active regions on the surface of the nucleus: In the prograde case, one region is close to the equator, and the others are at positive and negative mid-latitudes $\left(-45^{\circ}\right.$ for structure B and $+60^{\circ}$ for structure $\left.\mathrm{C}\right)$. Since a real topographic model of the surface does not exist, these locations cannot be constrained further. However, the emission direction of the jets cannot be more than $10^{\circ}$ away from our estimations or the simulations would differ significantly from the real images. We found that jets are best reproduced with particles between $10 \mu \mathrm{m}$ and $100 \mu \mathrm{m}$, and a reference velocity $v_{0}$ of $80 \mathrm{~m} \mathrm{~s}^{-1}$, which translates into a particle velocity in the range 30-45 $\mathrm{m} \mathrm{s}^{-1}$. At $1.4 \mathrm{AU}$, Tozzi et al. (2011) reported a typical grain size of several tens of $\mu \mathrm{m}$ and a velocity of $25-35 \mathrm{~m} \mathrm{~s}^{-1}$ for the average coma. We find that the velocity of dust particles in the jets is only slightly larger than in the rest of the coma, for a similar grain size. This is consistent with our understanding of the activity, where most of the outgassing and dust emission take place in a few selected regions that define the behavior of the whole coma. Figure 7 shows an example of our simulations for several dates.

Because our model reproduces the observations shortly before and after perihelion, we used it also to try to understand 


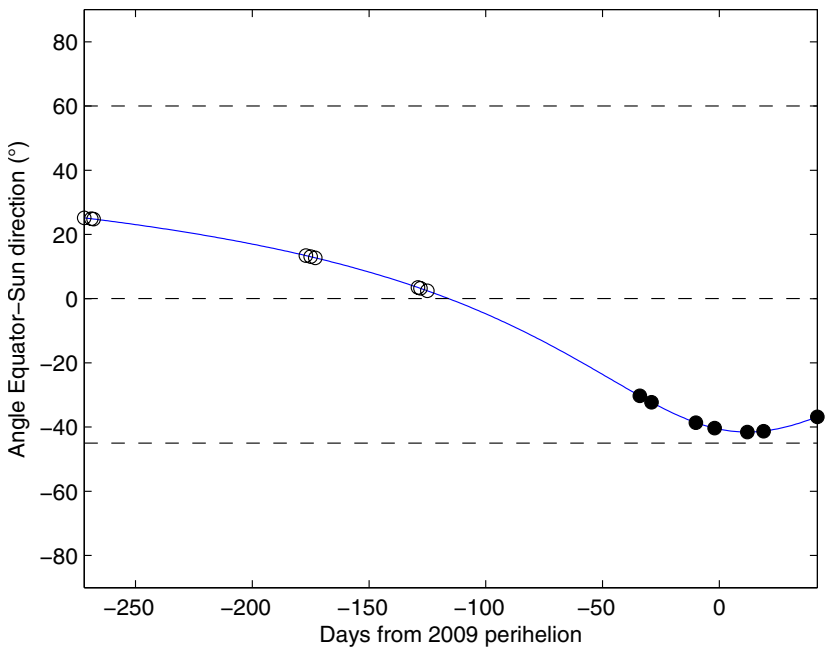

Fig. 8. Angle between the comet equator and the Sun direction in the epoch covered by our observations (01/06/2008 to 11/04/2009). The dashed lines indicate the estimated latitudes of the three active regions described in Sect. 4.2. Northern and southern hemisphere are defined as facing the ecliptic north and south. Circular markers indicate the epochs of our observations, empty circles represent images where no clear evidence of jets could be seen, whereas full markers indicate unambiguous detection.

whether the anisotropies detected in the coma between 3 and 2 AU before perihelion can be interpreted as dust structures or not. Figure 10 shows our results. we found that the same active regions could explain the detected features, provided that the dust particles emitted are small (maximum $10 \mu \mathrm{m}$ ), and have low velocities (typically $10-50 \mathrm{~m} \mathrm{~s}^{-1}$ ).

\subsection{Typical activity pattern for 67P}

We can summarize the two previous sections by giving a typical activity pattern for 67P. Figure 8 shows the calculated subsolar latitude during the epoch covered by our observations. As the comet approaches the Sun, its northern hemisphere (toward the ecliptic north) is illuminated while part of the southern one is in polar nights. During that period, no jets are visible. Activity in the equatorial region can exist but would be hidden in the dust tail due to the observing geometry. The subsolar point crosses the equator of the comet about 100 days before perihelion, and we start to detect jets emanating from the southern hemisphere roughly 30 days before perihelion. The weaker jet $C$ emanating from the northern hemisphere disappears about 30 days after perihelion.

From this point of view it looks as if the whole activity pattern can be explained by different illumination conditions according to the local season on the nucleus. The relative strength of the different jets can also be explained in the same way. We can reproduce the apparent weakness of jet $C$ if we assume the activity in this region to be present only during the day. When jets start to be detected, this part of the nucleus is illuminated only for a short time with respect to the others, at most $3 \mathrm{~h}$, and therefore will display less activity.

Figure 9 presents the typical nucleus illumination at perihelion for the ellipsoid model. There it can be seen that region $\mathrm{C}$ is poorly illuminated, if at all. This figure is of course not representative of the reality because the length of daytime at a specific place strongly depends on the topography. For this comet the jet activity can be explained by dayside activity alone, if the topography still allows some daylight in region $\mathrm{C}$ at perihelion. If not, the activity could be driven by the sublimation of $\mathrm{CO}_{2}$ ice

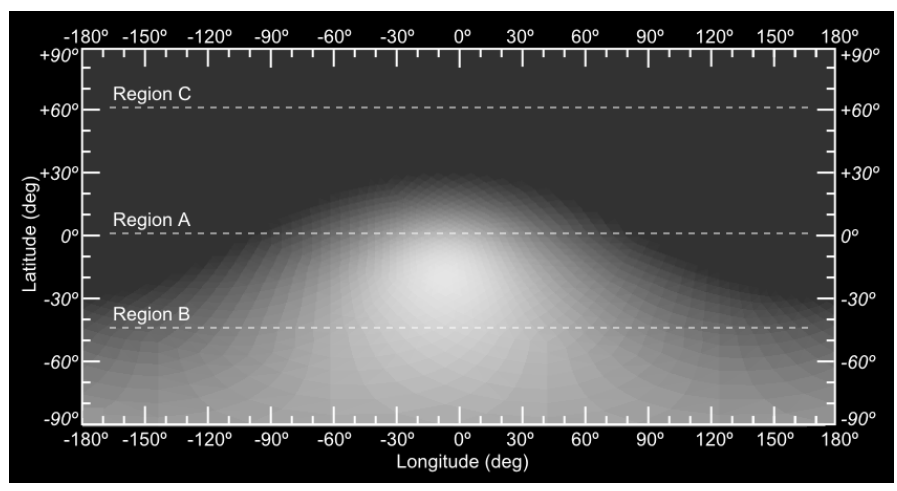

Fig. 9. Typical illumination of the nucleus in February 2009, at perihelion, calculated on the ellipsoid reference shape from Lamy et al. (2007). The dotted lines indicate the latitudes of active regions determined by COSSIM. The figure illustrates the clear difference in activity that can be expected between regions. For instance, one can see that region $\mathrm{B}$ is almost continuously illuminated, whereas region $\mathrm{C}$ is in polar winter at perihelion. Of course the real illumination of the nucleus depends strongly on the shape of the body and this picture cannot be fully representative of the local conditions.

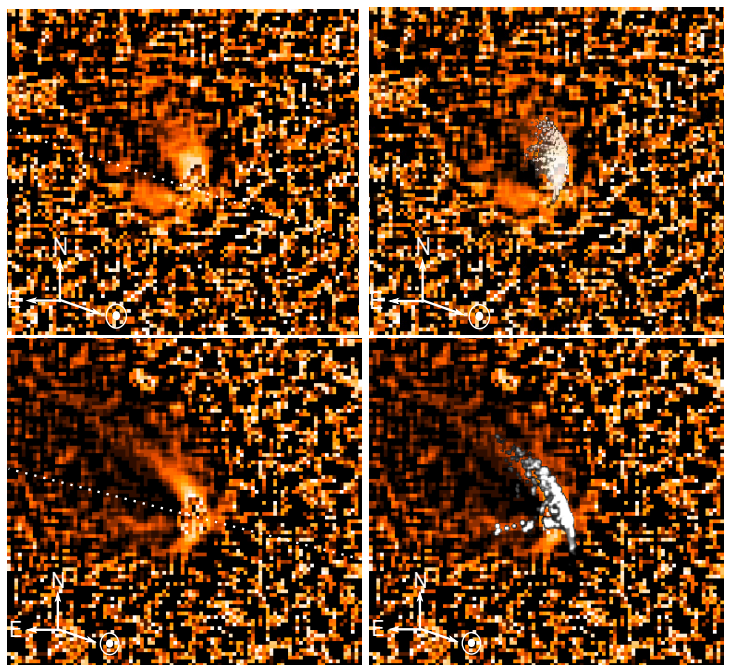

Fig. 10. Model of dust jets for 08 Sep. 2008 and 22 Oct. 2008. The left panels show Laplace-filtered images of 67P. the right panels show the same images with simulations overlaid. The agreement between model and detection suggests that some of detected structures are real and not artifacts created by the image enhancement technique. Other patterns present in the filtered images, in the sunward direction for all images and antisolar for Sep. 2008, cannot be reproduced by the model. We interpret them as the front edge of the dust coma, or the signature of the dust tail. The dotted line traces the orbital plane of the comet.

on the night side as was observed on 9P/Tempel 1 (Feaga et al. 2007; Vincent et al. 2010b).

The weakness of the jets in the pre-perihelion phase is also compatible with our model. As we have seen, most of the activity is located close to the equator or in the southern hemisphere, with only one active region in the north. This region should be emitting dust when illuminated, starting several months before perihelion; this is not the case. One possible explanation is that this region is partially depleted of volatile material, the ice being for instance buried under a deeper layer of dust than in other regions. It would then require the nucleus to be closer to the Sun for the sublimation to start. As we will see in the next section, this region was active at the time of the previous perihelion passage (2003) and might have lost most of its ices. This is a simplified scenario, however, and this discussion should be 
Table 2. Comparison between our model predictions of what the jet activity pattern of 67P should have been in March 2003 and published observations.

\begin{tabular}{lccc}
\hline \hline Structures & $A^{\circ}$ & $B^{\circ}$ & $C^{\circ}$ \\
\hline COSSIM prediction & 130 & 190 & 60 \\
Lara et al. (2005) & 130 & 195 & ND \\
Böhnhardt et al. (2005) & 125 & 190 & 66 \\
Weiler et al. (2004) & 130 & 190 & ND \\
\hline
\end{tabular}

Notes. Numbers refer to the projected position angle of the features, counted from the north. ND = structure not detected. Given the geometry of ground-based observations at that time, active region B will only produce one visible jet and not two as in 2009. Again region C is very poorly illuminated and jet $\mathrm{C}$ is predicted to be difficult to observe.

refined when realistic shape and thermal models for the comet are available.

\subsection{Comparison with the 2003 apparition}

In contrast to the other studies we performed on comets visited by spacecrafts, we do not yet have in situ data to validate our simulations. However, one simple test can be attempted. 67P has been observed for more than 15 years, and we have data covering three perihelion passages (1997, 2003, and 2009). 2003 is particularly interesting for us because the comet has been monitored in the same part of its orbit as in 2009, and observers reported several coma structures (Lara et al. 2005; Weiler et al. 2004; Böhnhardt 2005, unpublished, see Schulz et al. 2004, for reference of observations). If we assume that (a) the spin axis orientation did not change from 2003 to 2009 and (b) the regions active in 2009 were already active in 2003, our model derived from the 2009 data should describe the observation acquired in 2003. Assumption (a) is very realistic, indeed, we have shown in the previous section that the spin axis direction derived from the epoch when the comet was the most active is the same as the one derived from the inactive part of the orbit. Hence we do not expect the activity of previous perihelion passages to have modified the spin axis orientation by more than a few degrees. Assumption (b) must be considered more cautiously. There is no way to know if the same regions of the surface remain active from one apparition to the next. In fact, this depends on the source of activity. When the sublimating component is depleted, the region will become inactive, unless something triggers it again (impact or inner outburst exposing deeper layers to the Sun). Depending on the amount of material available for sublimation and on the sublimation rate, the same region can be active for several orbits, or fade away after one or more apparitions. That being said, one can remark that the activity of comet 9P/Tempel 1 has been observed at different epochs with two different spacecraft in the same regions of the surface (Farnham et al. 2012), so the assumption of longer lasting activity from the same area is not completely unrealistic.

Table 2 summarizes the results of our test. We simulated the dust jets created by the same active regions for the 2003 apparition with COSSIM, and compared this with observations performed by Lara et al. (2005) and Boehnhardt 2005 (unpublished, see Schulz et al. 2004, for reference of observations). Our simulation and the observations agree well. The mean of the difference between predicted and observed position angles is only $3.0^{\circ}$. One example image is shown in Fig. 11.

\subsection{Predictions for 2015}

Our model of activity for the comet is fully compatible with observations acquired in 2003 and 2009. We are now fairly
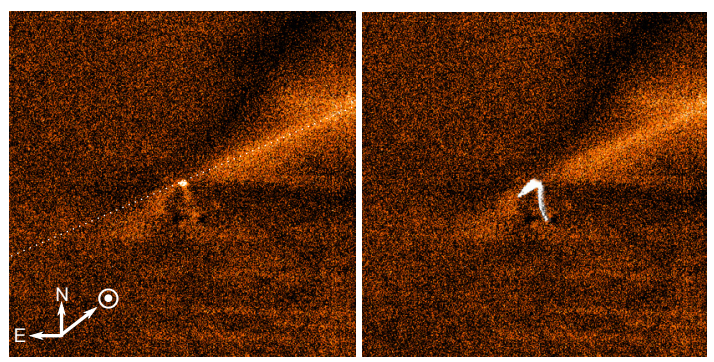

Fig. 11. Model of dust jets for 31 Mar. 2003. The left panel is broadband $R$ image of 67P acquired on 31 March 2003 (see Lara et al. 2005 for details). The right panel shows the same image with simulations overlaid. Field of view: $82 \times 82 \operatorname{arcsec}=163800 \mathrm{~km}$ at the comet. The dotted line traces the orbital plane of the comet.

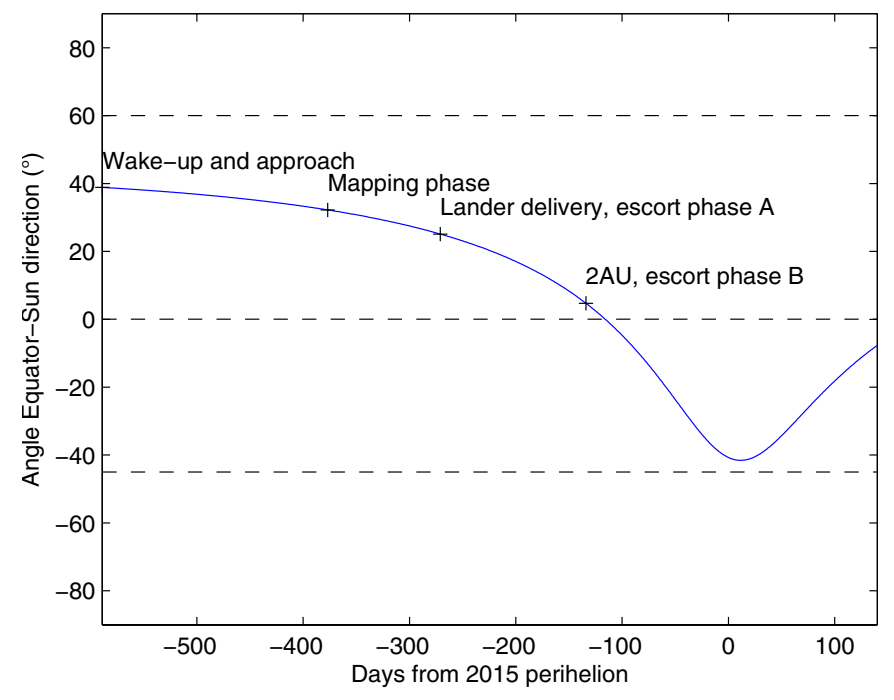

Fig. 12. Angle between the comet equator and the Sun direction in the epoch covered by the Rosetta mission (01/01/2014 to 31/12/2015). The dashed lines indicate the estimated latitudes of the three active regions described in Sect. 4.2. Northern and southern hemisphere are defined as facing the ecliptic north and south. Labels indicate the main different phases of the Rosetta mission.

confident that the same activity pattern will be repeated at the next perihelion passage in 2015 . That activity seems to rise after perihelion is advantageous for the safety of the spacecraft. The lander delivery is planned for November 2014, about a year before perihelion, which gives us plenty of time to fully characterize the nucleus. From geomorphology and surface composition we might be able to detect already which regions of the surface will become active, but we do not expect the larger jets to start before mid-2015. Of course, as better shape models will become available in the coming years, we will refine our predictions on the localization of the sources. Figure 12 shows the predicted subsolar latitude during the active phase of the Rosetta mission (from wake-up to end of mission). The pattern is very similar to that of $2008 / 2009$, with the Sun crossing the nucleus equator about 50 days before perihelion (13/08/2015). If the comet behaves as in 2003 and 2009, the main jets should become visible from Earth a month before perihelion, i.e., mid-July 2015. Figure 14 shows a simulation of the jets, with the same geometry as seen from Earth around the perihelion passage in 2015.

\section{Conclusions and outlook}

We have applied our numerical model of coma structures to 67P, prime target of the Rosetta mission. Based on data acquired 
Table 3. Summary of the results.

\begin{tabular}{llll}
\hline \hline Spin axis orientation: & $\mathrm{RA}=40 \pm 10^{\circ}$ & $\mathrm{Dec}=70 \pm 10^{\circ}$ & \\
Latitudes active regions: & $+60 \pm 10^{\circ}$, & $0 \pm 10^{\circ}$, & $-45 \pm 10^{\circ}$ \\
Typical grain size in the jets: & 10 to $100 \mu \mathrm{m}$ & & \\
Typical velocity: & 30 to $80 \mathrm{~m} \mathrm{~s}^{-1}$ & & \\
\hline
\end{tabular}

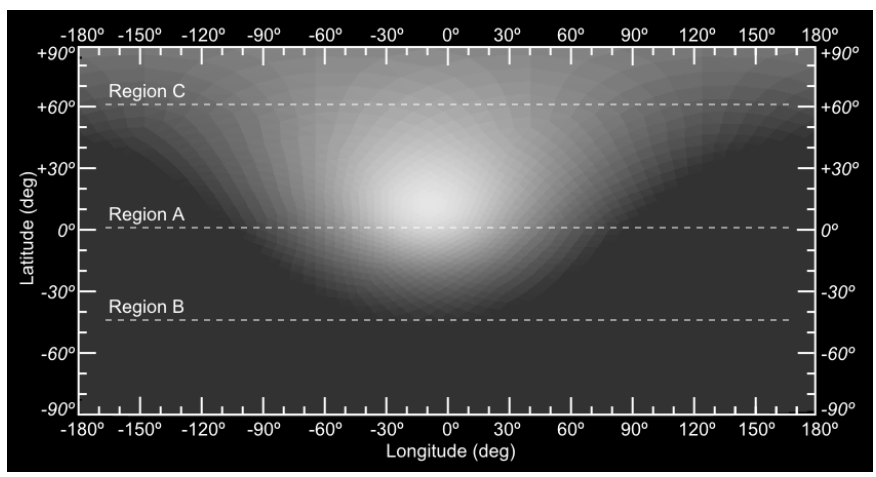

Fig. 13. Typical illumination of the nucleus during the lander delivery phase of the Rosetta mission (Nov. 2015), calculated on the ellipsoid reference shape from Lamy et al. (2007). The dotted lines indicate the latitudes of active regions determined by COSSIM. Depending on how different the real shape is from an ellipsoid local illumination conditions will vary, but overall the northern hemisphere will be the most illuminated at that time.

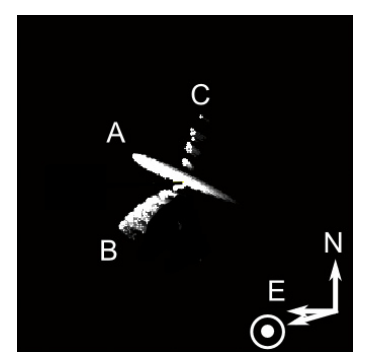

Fig. 14. Simulation of the jets created by the three main active regions of 67P at perihelion 2015, as seen from Earth. This image shows the position of dust particles in the plane of sky. The model assumes no significant change in spin axis orientation and location of active areas on the surface of the nucleus with respect to previous perihelion passages. Note that due to the observing geometry region "B" will create a single jet and not a double feature like the one seen in 2009. As explained in 4.3, region "C" seems to weaken from 2003 to 2004 . This is possibly due to a depletion of volatile elements in this area of the nucleus. Therefore there is a possibility that a jet from this region might be too weak to be observable from Earth in 2015.

during the 2008-2009 apparition, we determined the spin axis orientation and found a value of $\mathrm{RA}=40^{\circ}$ and $\mathrm{Dec}=70^{\circ}$ in agreement with existing determinations. This supports the idea that $67 \mathrm{P}$ is in a stable state of rotation; its spin axis orientation has not significantly changed over the last two orbits, at least down to the resolution of our observations. Our numerical model adequately simulated the jet geometry observed and the global activity pattern, leading to a determination of the localization of at least three active sources on the surface of the nucleus. We estimated the activity to take place at the equator and mid latitudes, with an emphasis on the hemisphere illuminated around perihelion. All results are summarized in Table 3. Using the parameters derived from 2009, we were able to reconstruct the activity of the previous apparition (2003), which indicates that the same regions of the surface were already active at that time. We investigated here the largest sources of activity. While they create the jet structures observed in the coma, they only represent a fraction of the total brightness and one must assume that there are many other small sources of activity distributed all over the surface, which cannot be detected directly from ground-based observations. It is also very likely than some of the studied jets are not single features but result of a number of small jets in a given region, whose emissions are seen as a single large source. Only in-situ observations will be able to constrain the analysis further.

We used and simulated ground-based data only but we are now moving toward the understanding and simulation of in situ images. As a support to the Rosetta mission, we are improving COSSIM, and the new version will describe the physics of gas/dust interaction very close to the surface in detail, taking into account the complex topography of the nucleus.

Acknowledgements. Jean-Baptiste Vincent thanks Hermann Böhnhardt for his help on the data analysis and the final review of the paper. L. M. Lara's work has been funded by the research project AyA 2009-08011 awarded by the Spanish Ministerio de Ciencia e Innovacion. This research has made use of NASA's Astrophysics Data System Bibliographic Services.

\section{References}

A'Hearn, M. F., Hoban, S., Birch, P. V., et al. 1986, Nature, 324, 649

Beisser, K., \& Drechsel, H. 1992, Ap\&SS, 191, 1

Belton, M. J. S. 2010, Icarus, 210, 881

Boehnhardt, H., \& Birkle, K. 1994, A\&AS, 107, 101

Burns, J. A., Lamy, P. L., \& Soter, S. 1979, Icarus, 40, 1

Chesley, S. R. 2004, in AAS/Division for Planetary Sciences Meeting Abstracts \#36, BAAS, 36, 1118

Crifo, J. F., \& Rodionov, A. V. 1997, Icarus, 129, 72

Davidsson, B. J. R., \& Gutiérrez, P. J. 2005, Icarus, 176, 453

Farnham, T. L., Wellnitz, D. D., Hampton, D. L., et al. 2007, Icarus, 187, 26

Farnham, T. L., Bodewits, D., Li, J.-Y., et al. 2012, Icarus, submitted

Feaga, L. M., A'Hearn, M. F., Sunshine, J. M., Groussin, O., \& Farnham, T. L. 2007, Icarus, 190, 345

Finson, M. L., \& Probstein, R. F. 1968, ApJ, 154, 353

Fulle, M. 1987, A\&A, 171, 327

Fulle, M. 1999, Adv. Space Res., 24, 1087

Lamy, P. L., Toth, I., Weaver, H. A., et al. 2006, A\&A, 458, 669

Lamy, P. L., Toth, I., Davidsson, B. J. R., et al. 2007, Space Sci. Rev., 128, 23

Lara, L. M., de León, J., Licandro, J., \& Gutiérrez, P. J. 2005, Earth Moon Planets, 97, 165

Lara, L. M., Lin, Z.-Y., Rodrigo, R., \& Ip, W.-H. 2011, A\&A, 525, A36

Larson, S. M., \& Sekanina, Z. 1984, AJ, 89, 571

Larson, S. M., \& Slaughter, C. 1992, Asteroids, Comets, Meteors 1991, 337

Lin, Z.-Y., Lara, L. M., Vincent, J.-B., \& Ip, W.-H. 2012, A\&A, 537, A101

Markovich, M. Z., \& Markovich, N. M. 2001, Solar System Research, 35, 320

Min, M., Hovenier, J. W., de Koter, A., Waters, L. B. F. M., \& Dominik, C. 2005 Icarus, 179,158

Samarasinha, N. H., \& Larson, S. M. 2011, in EPSC/DPS joint meeting

Schleicher, D. G. 2006, Icarus, 181, 442

Schleicher, D. G., \& Farnham, T. L. 2004, Photometry and imaging of the coma with narrowband filters, eds. M. C. Festou, H. U. Keller, \& H. A. Weaver, 449

Schulz, R., Stüwe, J. A., \& Böhnhardt, H. 2004, A\&A, 422, L19

Schwarz, G., Cosmovici, C., Mack, P., \& Ip, W. 1989, Adv. Space Res., 9, 217

Sekanina, Z. 1987, ESA SP-278, 315

Szegö, K., Crifo, J.-F., Rodionov, A. V., \& Fulle, M. 2002, Earth Moon Planets, 90, 435

Tozzi, G. P., Patriarchi, P., Boehnhardt, H., et al. 2011, A\&A, 531, A54

Vincent, J.-B. 2010, Ph.D. thesis (uni-edition ISBN 978-3-942171-31-1)

Vincent, J.-B., Böhnhardt, H., Bertini, I., et al. 2010a, Earth Moon Planets, 106, 27

Vincent, J.-B., Böhnhardt, H., \& Lara, L. M. 2010b, A\&A, 512, A60

Vincent, J.-B., Sierks, H., Rose, M., \& Skorov, Y. 2012, in EPSC 2012, 358

Weiler, M., Rauer, H., \& Helbert, J. 2004, A\&A, 414, 749 